\title{
Avaliação de área recuperada sobre cava de areia em São Paulo, SP, Brasil
}

\author{
Daiana Aparecida Damião Gonzalo ${ }^{1}$, Ricardo José Francischetti Garcia² e Eduardo Pereira Cabral Gomes ${ }^{3,4}$
}

Recebido: 4.05.2015; aceito: 17.09.2015

\begin{abstract}
Evaluation of a reclaimed area over sand mining in São Paulo, São Paulo State, Brazil). Mining is a fundamental economic activity that sustains basic sectors. In abandoned sand mines, the natural vegetation recovery is very slow and in many cases, the recolonization may take decades before the organic material and the soil structure are restored. To minimize this impact, the law requires restoration of native vegetation. In this paper, we evaluate the structure of the vegetation after nine years of planting in an area degraded by sand mining. We recorded which of the species better established in the area. All tree individuals with $\mathrm{DBH}>2.0 \mathrm{~cm}$ had DBH and height recorded and were identified. The number of species was slightly higher (7.5\%) than the number used in planting. Over $70 \%$ of those are early successional species. Four species showed individuals over $6 \mathrm{~m}$ in height after nine years of planting and are recommended for future recovery plantations. The most successful species was of the early stages of succession.
\end{abstract}

Keywords: mining, native species, restoration

RESUMO - (Avaliação de área recuperada sobre cava de areia em São Paulo, SP, Brasil). A mineração é uma atividade econômica fundamental para vários setores. Nas minas de areia, a recuperação natural da vegetação é lenta e o processo de sucessão pode retardar por décadas até que a matéria orgânica e a estrutura do solo sejam restabelecidas. Para minimizar este impacto a legislação exige a restauração da vegetação nativa. Neste trabalho avaliamos a estrutura da vegetação após nove anos de plantio em área degradada por mineração de areia. Procuramos determinar quais as espécies plantadas que se estabeleceram melhor na área. Na amostragem foram determinados altura e diâmetro, e foram identificados todos os indivíduos arbóreos com DAP $>2 \mathrm{~cm}$. O número de espécies registradas foi 7,5\% superior ao utilizado no plantio. Mais de 70\% dos indivíduos são de espécies dos estágios iniciais de sucessão. Quatro espécies apresentaram indivíduos com mais de $6 \mathrm{~m}$ de altura após os nove anos de plantio e são recomendadas para futuros plantios de recuperação.

Palavras-chave: espécies nativas, mineração, restauração ecológica

\section{Introdução}

A mineração é caracterizada como uma atividade econômica fundamental que sustenta setores básicos do país como a agricultura, a construção civil e as indústrias cerâmicas, cimenteira e vidreira. Nas minas de areia, a extração consiste no decapeamento do solo, seguido por lavra de desmonte hidráulico, da qual os rejeitos são depositados em bacias de decantação (Lelles et al. 2005, Padmalal \& Maya 2014). Para o Estado de São Paulo, a importância da mineração de areia pode ser constatada por ter representado $41,8 \%$ do valor e $51,5 \%$ da tonelagem totais mineradas em fins da década passada (Cabral Junior et al. 2008).
Os impactos ambientais dessa atividade são grandes (Knapik \& Marinho 2008, Nobre Filho et al. 2011, Devide et al. 2014) e a recuperação natural da vegetação é muito lenta pela falta de solo adequado. Em muitos casos, o processo de sucessão assistido pode se deter por décadas antes que a matéria orgânica e a estrutura do solo sejam restabelecidas (Almeida \& Sanchez 2005). Para minimizar este impacto a legislação exige e orienta a restauração da vegetação nativa nestes locais pela apresentação e execução de um Plano de Recuperação de Área Degradada (PRAD). O monitoramento destes, no entanto, tem se mostrado insuficiente, ou na melhor das situações, limitado apenas aos primeiros anos após a implantação

1. Universidade de Santo Amaro, Rua Isabel Schmidt, 349 - Santo Amaro, 04743-030, São Paulo, SP, Brasil

2. Prefeitura Municipal de São Paulo, Departamento de Parques e Áreas Verdes, Av. IV Centenário, 1268 Parque Ibirapuera, portão 7 A, Herbário Municipal Ibirapuera, 04030-000 São Paulo, SP, Brasil

3. Instituto de Botânica, Núcleo de Pesquisa em Ecologia, Av. Miguel Estefano 3687, Caixa Postal 68041, 04045-972, São Paulo, SP, Brasil

4. Autor para correspondência: eduardo.pcgomes@pq.cnpq.br 
com a quantificação de mudas mortas e a respectiva substituição (Bittar 1997, Mechi \& Sanches 2010).

A efetiva recuperação de áreas de explorações é controversa como em todo tema de restauração ecológica de florestas nativas (Brancalion et al. 2010, Durigan et al. 2010, Gomes et al. 2013). Para as áreas de mineração de areia, estudos têm mostrado que várias ações de restauração tem tido alcance limitado (Bittar 1997) ou mesmo apresentado resultados piores do que o simples abandono da área (Pinheiro et al. 2008). Em muitos casos a recuperação limitou-se a atenuar o impacto visual (Bittar 2000) e o resultado da restauração é ruim ou regular para a maioria das áreas mineradas (São Paulo 2008).

Neste trabalho avaliamos a estrutura da vegetação após nove anos de plantio em área degradada por mineração de areia no município de São Paulo, SP, Brasil. Procuramos determinar quais das espécies utilizadas se estabeleceram melhor (maior proporção de sobreviventes) na área e quais apresentaram maior crescimento após o período.

\section{Material e métodos}

A área da Mineradora Viterbo Machado Luz, uma antiga cava de extração de areia, situa-se em Parelheiros, zona Sul do Município de São Paulo, SP, Brasil em uma região de mananciais inserida na Área de Proteção Ambiental (APA) Bororé Colônia (Braga 2005). A área avaliada, denominada AR5, possui aproximadamente $1.100 \mathrm{~m}^{2}$ de área minerada (figura 1).

O plantio de restauração foi realizado em fins de 1999, nove anos antes da avaliação ser realizada. No local foram plantadas espécies arbóreas pioneiras e secundárias iniciais em covas de $0,60 \times 0,60 \times 0,60 \mathrm{~cm}$, com espaçamento de $3 \times 2,5 \mathrm{~m}$, no total de 146 mudas (Braga 2005). O modelo adotado foi o de consorciamento de espécies nativas dos estágios sucessionais pioneiro, secundário e climácico, utilizando-se mudas entre 30 e $60 \mathrm{~cm}$ de altura. As mudas das 28 espécies (tabela 1) foram separadas apenas por grupo ecológico, alternando-se nas linhas os plantios de pioneiras com secundárias ou climácicas, distribuindo-se as espécies ao acaso no momento do plantio. Semestralmente, nos primeiros dois anos, foi feito o coroamento de manutenção ao redor das mudas.

A avaliação do plantio foi feita em 12 parcelas de $6 \times 15 \mathrm{~m}$ (total de $1.080 \mathrm{~m}^{2}$ ) que cobriram toda a área plantada. Nestas unidades amostrais foram incluídos todos os indivíduos com caule de diâmetro a $1,30 \mathrm{~cm}$ de altura do solo (DAP) igual ou superior a $2 \mathrm{~cm}$, inclusive mortos. A altura das plantas foi obtida utilizando uma vara de seis metros graduada a cada meio metro. Materiais vegetativo e reprodutivo foram coletados e identificados no Herbário Municipal de São Paulo (PMSP) com ajuda de especialistas. Foram realizados os procedimentos de coleta, preparo e acondicionamento de material botânico conforme Fidalgo \& Bononi (1989). Os cálculos dos descritores quantitativos de densidade, dominância por área basal e frequência foram feitos segundo Mueller-Dombois \& Ellenberg (1974).

A listagem dos nomes das espécies foi atualizada de acordo com o proposto por Angiosperm Phylogeny Group - APG III (APG III 2009). A validade da nomenclatura das espécies foi conferida pela Lista de Espécies da Flora do Brasil 2010 (Forzza et al. 2010) e pelo checklist para a Flora do Estado de São Paulo (Wanderley et al. 2011); em caso de conflito prevaleceu a primeira.

\section{Resultados}

Foram amostrados 219 indivíduos de 30 espécies, pertencentes a 12 famílias e a 25 gêneros (tabela 2) resultando em uma densidade de 2.027,7 indivíduos $\mathrm{ha}^{-1}$ e um incremento de $50 \%$ do número de indivíduos em relação ao plantio. A altura máxima obtida foi de 13,5 m e o diâmetro máximo encontrado foi de $26,5 \mathrm{~cm}$. Nove anos após o plantio, a área basal total atingiu $1,86 \mathrm{~m}^{2}\left(17,22 \mathrm{~m}^{2} \mathrm{ha}^{-1}\right)$. Considerando

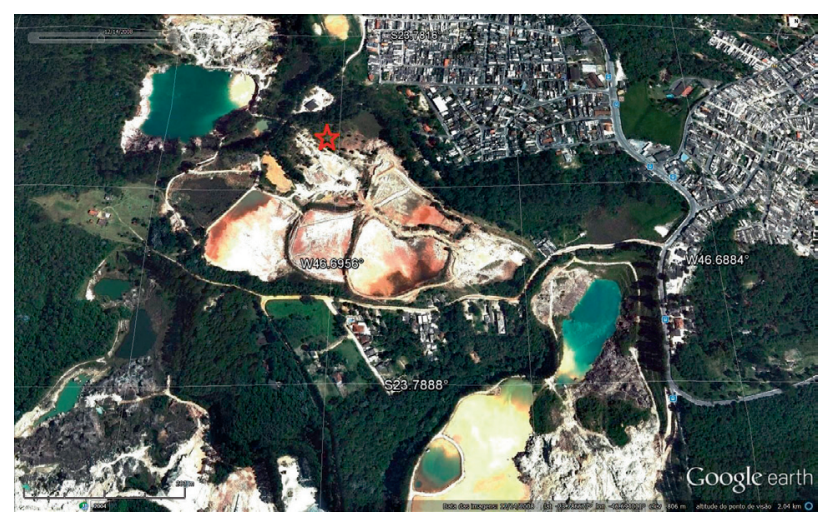

Figura 1. Vista aérea da Mineradora Viterbo Machado, São Paulo, SP, Brasil. $\imath^{2}$ - Área de estudo. Fonte: Google Earth.

Figure 1. Aerial view of Viterbo Machado Luz Mining, São Paulo, São Paulo State, Brasil. $\hat{\imath}$ - Study area. Source: Google Earth. 
apenas os indivíduos com DAP $>4,7 \mathrm{~cm}$, utilizado na maioria dos levantamentos de vegetação natural no Estado de São Paulo, a densidade foi de 1.240,7 indivíduos $\mathrm{ha}^{-1} \mathrm{e}$ a área basal $16,98 \mathrm{~m}^{2} \mathrm{ha}^{-1}$. A distribuição de diâmetros seguiu a típica forma de $\mathrm{J}$ invertido (figura 2) indicando o estoque de jovens regenerantes.

Treze das 28 espécies $(46,4 \%)$ do plantio inicial não foram registradas nove anos depois (tabela 1). Por outro lado, dentre as registrados no presente estudo, 10 espécies não constam do plano inicial de recuperação (tabela 2). Das 30 espécies amostradas, sete (Citrus $\times$ limon (L.) Burm.f., Eryobothria japonica (Thunb.) Lindl., Erythrina speciosa Andrews, Eucalyptus sp., Jacaranda mimosifolia D.Don., Petrea volubilis L. e Psidium guajava L.) não apresentam distribuição original na região, sendo três destas oriundas de outros continentes (C. limon, E. japonica e Eucalyptus sp.); estas espécies contribuíram com $19,2 \%$ da densidade total.

Quatro espécies tiveram indivíduos que atingiram ou ultrapassaram 10 metros de altura (figura 3), o guapuruvu (Schizolobium parahyba (Vell.) S.F.Blake), o ipê (Handroanthus impetiginosus (Mart. ex DC.)

Tabela 1. Lista das 28 espécies utilizadas para a recuperação da área AR5, na Mineradora Viterbo Machado Luz, São Paulo, SP, Brasil. *espécies não encontradas na amostragem; **provável erro na identificação. Fonte: Braga (2005).

Table 1. List of 28 species used for the recovery of the AR5 area in Viterbo Machado Luz Mining, São Paulo State, Brazil. *species not found in sampling; **probably misidentification. Source: Braga (2005).

\begin{tabular}{|c|c|}
\hline Família & Espécie \\
\hline \multirow[t]{2}{*}{ Anacardiaceae } & Lithraea molleoides (Vell.) Engl.* \\
\hline & Schinus terebinthifolius Raddi \\
\hline Apocynaceae & Aspidosperma olivaceum Müll.Arg.** \\
\hline Arecaceae & Euterpe edulis Mart.* \\
\hline \multirow[t]{4}{*}{ Bignoniaceae } & Handroanthus impetiginosus (Mart. ex DC.) Mattos \\
\hline & Handroanthus chrysotrichus Toledo \\
\hline & Jacaranda mimosifolia D.Don. \\
\hline & Jacaranda puberula Cham.* \\
\hline Boraginaceae & Cordia trichotoma (Vell.) Arráb. ex Steud.* \\
\hline Euphorbiaceae & Croton urucurana Baill.* \\
\hline \multirow[t]{8}{*}{ Fabaceae } & Anadenanthera peregrina (L.) Speg.* \\
\hline & Cyclolobium brasiliense Benth.* \\
\hline & Enterolobium contortisiliquum (Vell.) Morong* \\
\hline & Erythrina speciosa Andrews \\
\hline & Hymenaea courbaril L. \\
\hline & Inga sp. \\
\hline & Lonchocarpus cultratus (Vell.) Az.-Tozzi \& H.C.Lima* \\
\hline & Peltophorum dubium (Spreng.) Taub. \\
\hline \multirow[t]{4}{*}{ Malvaceae } & Ceiba speciosa (A.St.-Hil.) Ravenna \\
\hline & Guazuma crinita Mart.** \\
\hline & Guazuma ulmifolia Lam. \\
\hline & Luehea sp.** \\
\hline Moraceae & Ficus sp.* \\
\hline \multirow[t]{3}{*}{ Myrtaceae } & Plinia cauliflora (DC.) Kausel* \\
\hline & Psidium cattleianum Sabine \\
\hline & Psidium guajava $\mathrm{L}$. \\
\hline Rutaceae & Esenbeckia leiocarpa Engl.* \\
\hline Verbenaceae & Citharexyllum myrianthum* \\
\hline
\end{tabular}


Tabela 2. Espécies, respectivas famílias e descritores quantitativos, área da Mineradora Viterbo Machado Luz, São Paulo, SP, Brasil. Ab: abreviatura; Ni: número de indivíduos; F: número de parcelas em que a espécie ocorreu; $\mathrm{m}^{2} \mathrm{ha}^{-1}$ : área basal; DR: densidade relativa; FR: frequência relativa; DoR: Dominância Relativa; VC: Valor de Importância (DR + DoR). *espécies que não ocorrem naturalmente na região.

Table 2. Species, families, and quantitative descriptors. Viterbo Machado Luz Mining, São Paulo, São Paulo State, Brazil. Ab: abbreviation; Ni: number of individuals; F: number of plots in which the species occurred; $\mathrm{m}^{2} \mathrm{ha}^{-1}$ : basal area; DR: relative density; FR: relative frequency; DoR: relative dominance; VC: cover value (DR + DoR). *species that do not occur in theregion.

\begin{tabular}{|c|c|c|c|c|c|c|c|c|}
\hline Espécie (Família) & $\mathrm{Ab}$ & $\mathrm{Ni}$ & $\mathrm{F}$ & $\begin{array}{c}\mathrm{AB} \\
\mathrm{m}^{2} \mathrm{ha}^{-1}\end{array}$ & $\begin{array}{c}\mathrm{DR} \\
\%\end{array}$ & $\begin{array}{c}\text { FR } \\
\%\end{array}$ & $\begin{array}{c}\text { DoR } \\
\%\end{array}$ & $\begin{array}{l}\mathrm{VC} \\
\%\end{array}$ \\
\hline Schizolobium parahyba (Vell.) S.F.Blake (Fabaceae) & $\mathrm{Sp}$ & 29 & 12 & 0,667 & 13,2 & 10,7 & 36,6 & 49,8 \\
\hline Erythrina speciosa Andrews (Fabaceae)* & Es & 21 & 8 & 0,270 & 9,5 & 7,1 & 14,8 & 24,4 \\
\hline Psidium sp. (Myrtaceae) & $\mathrm{P}$ & 28 & 10 & 0,105 & 12,7 & 8,9 & 5,8 & 18,5 \\
\hline Psidium cattleianum Sabine (Myrtaceae) & Ps & 23 & 9 & 0,103 & 10,5 & 8,0 & 5,7 & 16,1 \\
\hline Ceiba speciosa (A.St.-Hil.) Ravenna & Cs & 11 & 6 & 0,201 & 5,0 & 5,4 & 11,0 & 16,0 \\
\hline Hymenaea courbaril L. (Fabaceae) & $\mathrm{Hc}$ & 19 & 10 & 0,122 & 8,6 & 8,9 & 6,7 & 15,3 \\
\hline Petrea volubilis L. (Verbenaceae)* & $\mathrm{Pv}$ & 10 & 6 & 0,129 & 4,5 & 5,4 & 7,1 & 11,6 \\
\hline Inga sp. (Fabaceae) & I & 20 & 7 & 0,032 & 9,1 & 6,3 & 1,8 & 10,9 \\
\hline Eugenia uniflora L. (Myrtaceae) & $\mathrm{Eu}$ & 7 & 4 & 0,013 & 3,2 & 3,6 & 0,7 & 3,9 \\
\hline Eryobothria japonica (Thunb.) Lindl. (Rosaceae)* & $\mathrm{Ej}$ & 3 & 1 & 0,046 & 1,4 & 0,9 & 2,5 & 3,9 \\
\hline Cedrela fissilis Vell. (Meliaceae) & $\mathrm{Cf}$ & 3 & 2 & 0,041 & 1,4 & 1,8 & 2,2 & 3,6 \\
\hline Psidium guajava L. (Myrtaceae)* & $\mathrm{Pg}$ & 5 & 3 & 0,022 & 2,3 & 2,7 & 1,2 & 3,5 \\
\hline $\begin{array}{l}\text { Handroanthus chrysotrichus (Mart. ex A.DC.) Mattos } \\
\text { (Bignoniaceae) }\end{array}$ & $\mathrm{Hc}$ & 4 & 2 & 0,030 & 1,8 & 1,8 & 1,6 & 3,5 \\
\hline Luehea divaricata Mart. (Malvaceae) & $\mathrm{Ld}$ & 6 & 4 & 0,001 & 2,7 & 3,6 & 0,1 & 2,7 \\
\hline Tibouchina granulosa Cogn (Melastomaceae) & $\operatorname{Tg}$ & 4 & 3 & 0,001 & 1,8 & 2,7 & 0,0 & 1,8 \\
\hline $\begin{array}{l}\text { Handroanthus impetiginosus (Mart. ex DC.) Mattos } \\
\text { (Bignoniaceae) }\end{array}$ & $\mathrm{Hi}$ & 3 & 3 & 0,008 & 1,4 & 2,7 & 0,4 & 1,8 \\
\hline Indeterminada & & 3 & 3 & 0,003 & 1,4 & 2,7 & 0,2 & 1,5 \\
\hline Guazuma ulmifolia Lam. (Malvaceae) & $\mathrm{Gu}$ & 3 & 2 & 0,001 & 1,4 & 1,8 & 0,1 & 1,4 \\
\hline Tibouchina sp. (Melastomaceae) & $\mathrm{T}$ & 3 & 3 & 0,001 & 1,4 & 2,7 & 0,1 & 1,4 \\
\hline Aspidosperma sp. (Apocynaceae) & A & 2 & 1 & 0,041 & 0,9 & 0,9 & 0,2 & 1,2 \\
\hline Citrus x limon (L.) Burm.f. (Rutaceae)* & $\mathrm{Cl}$ & 2 & 2 & 0,004 & 0,9 & 1,8 & 0,2 & 1,1 \\
\hline Schinus terebinthifolius Raddi (Anacardiaceae) & $\mathrm{St}$ & 1 & 1 & 0,005 & 0,5 & 0,9 & 0,3 & 0,8 \\
\hline Handroanthus heptaphyllus (Martius) Mattos (Bignoniaceae) & $\mathrm{Hh}$ & 1 & 1 & 0,004 & 0,5 & 0,9 & 0,2 & 0,7 \\
\hline Malpighia glabra L. (Malpighiaceae) & $\mathrm{Mg}$ & 1 & 1 & 0,003 & 0,5 & 0,9 & 0,2 & 0,6 \\
\hline Peltophorum dubium (Spreng.) Taub. (Fabaceae) & $\mathrm{Pd}$ & 1 & 1 & 0,002 & 0,5 & 0,9 & 0,1 & 0,6 \\
\hline Eucalyptus sp. (Myrtaceae)* & $\mathrm{E}$ & 1 & 1 & 0,002 & 0,5 & 0,9 & 0,1 & 0,6 \\
\hline Jacaranda mimosifolia D.Don. (Bignoniaceae)* & $\mathrm{Jm}$ & 1 & 1 & 0,002 & 0,5 & 0,9 & 0,1 & 0,5 \\
\hline Myroxylon peruiferum L.f. (Fabaceae) & $\mathrm{Mp}$ & 1 & 1 & 0,002 & 0,5 & 0,9 & 0,1 & 0,5 \\
\hline Mimosa caesalpiniifolia Benth. (Fabaceae) & $\mathrm{Mc}$ & 1 & 1 & 0,001 & 0,5 & 0,9 & 0,1 & 0,5 \\
\hline Tipuana tipu (Benth.) Kuntze (Fabaceae)* & $\mathrm{Tt}$ & 1 & 1 & 0,001 & 0,5 & 0,9 & 0,1 & 0,5 \\
\hline Luehea grandiflora Mart. \& Zucc. (Malvaceae) & $\mathrm{Lg}$ & 1 & 1 & 0,001 & 0,5 & 0,9 & 0,1 & 0,5 \\
\hline Total & & 219 & 111 & 1,860 & 100,0 & 100,0 & 100,0 & 200,0 \\
\hline
\end{tabular}


Mattos), o cedro-rosa (Cedrela fissilis Vell.) e a florde-são-miguel (Petrea volubilis L.). A última é uma espécie semi-escandente com origem no México. Além destas, outras três espécies tiveram uma altura média superior a seis metros (figura 3), Luehea divaricata Mart., Guazuma ulmifolia Lam. e Tibouchina sp. A maioria dos indivíduos (51\%) não ultrapassou quatro metros de altura.

\section{Discussão}

A realização de estudos sobre a estrutura de populações de espécies colonizadoras de áreas fortemente perturbadas é uma ferramenta importante a ser utilizada para definir estratégias de reabilitação e recuperação (Nappo et al. 2004). Desta forma, a fitossociologia permite analisar o desempenho de cada espécie, dado fundamental no planejamento de recuperação de áreas degradadas (Rodrigues \& Gandolfi 1998).

Houve grandes diferenças entre as espécies tanto em relação ao crescimento quanto ao estabelecimento. Para este último parâmetro, a falta do número exato de mudas plantadas para cada espécie não permitiu uma quantificação precisa da sobrevivência até o recenso. O que pode ser observado é a ausência de quase a metade das espécies inicialmente plantadas e o registro de quase um terço das espécies $(31,8 \%)$ como regenerantes espontâneas, ou seja, ausentes do plantio inicial. Parte da diferença pode ser devida a erros na identificação inicial das mudas de Aspidosperma olivaceum Müll.Arg. e Luehea sp. A

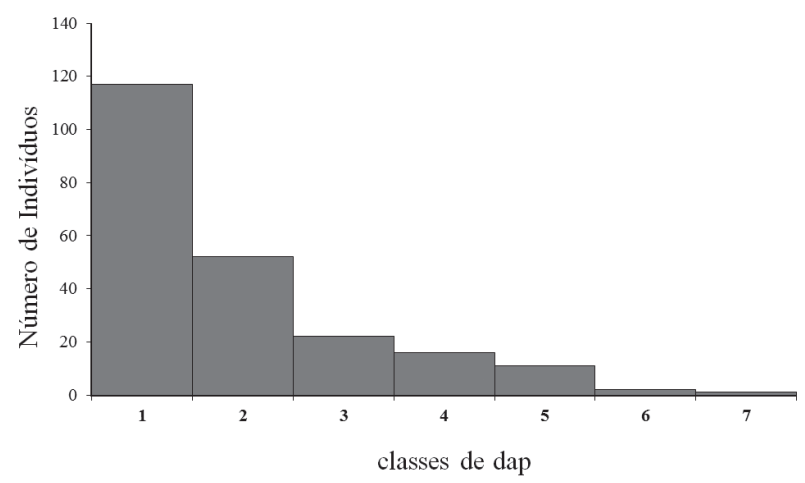

Figura 2. Distribuição diamétrica dos indivíduos arbóreos amostrados na área da Mineradora Viterbo Machado Luz, São Paulo, SP, Brasil. Classes: $1=2 \mathrm{~cm} \leq$ DAP $<6 \mathrm{~cm} ; 2=6 \leq$ DAP $<10,0 \mathrm{~cm} ; 3=10 \mathrm{~cm} \leq \mathrm{DAP}<14,0 \mathrm{~cm} ; \ldots \ldots . . ; 7=26,0 \leq \mathrm{DAP}$ $\leq 30,0 \mathrm{~cm}$.

Figure 2. Distribution of DBH in tree community; DBH classes: Classes: $1=2 \mathrm{~cm} \leq \mathrm{DBH}<6 \mathrm{~cm} ; 2=6 \leq \mathrm{DBH}<10,0 \mathrm{~cm} ; 3=10$ $\mathrm{cm} \leq \mathrm{DBH}<14,0 \mathrm{~cm} ; \ldots \ldots . ; 7=26,0 \leq \mathrm{DBH} \leq 30,0 \mathrm{~cm}$. primeira pode ser o indivíduo por nós identificado como Aspidosperma sp. e a última pode vir a reunir as duas espécies de Luehea identificadas. Para as demais espécies é improvável que erros de identificação possam ter ocorrido. Cabe destacar que somente $1,8 \%$ dos indivíduos amostrados tiveram menos de dois metros de altura e muitas das mudas inicialmente plantadas podem ter sobrevivido, mas não atingiram o critério mínimo de inclusão aqui utilizado. Este pode ser o caso de Euterpe edulis Mart., Plinia cauliflora (DC.) Kausel e, possivelmente, de Cordia trichotoma (Vell.) Arrab. ex Steud. e Esenbeckia leiocarpa Engl.

Da composição florística também cabe observar a presença de sete espécies não nativas regionalmente que contribuíram com mais de $10 \%$ do total de indivíduos amostrados. A invasão de espécies exóticas é considerada a segunda maior causa de extinção, logo após a perda de habitat (Richardson et al. 2000). Ainda que em alguns estudos tenha sido observado que espécies exóticas apresentaram o melhor resultado na recuperação de áreas (Souza et al. 2001, Marinho et al. 2004), a ausência de controle das espécies exóticas pode vir a comprometer todo o processo de recuperação mesmo que, em uma primeira etapa, um mínimo de estrutura e funções tenham sido restabelecidos, como o controle de erosão.

Dentre os padrões estruturais, após nove anos, os valores de área basal se aproximaram daqueles registrados para algumas matas nativas da região da Grande São Paulo (Ogata \& Gomes 2006). Dos vinte e um trabalhos analisados por Ogata \& Gomes

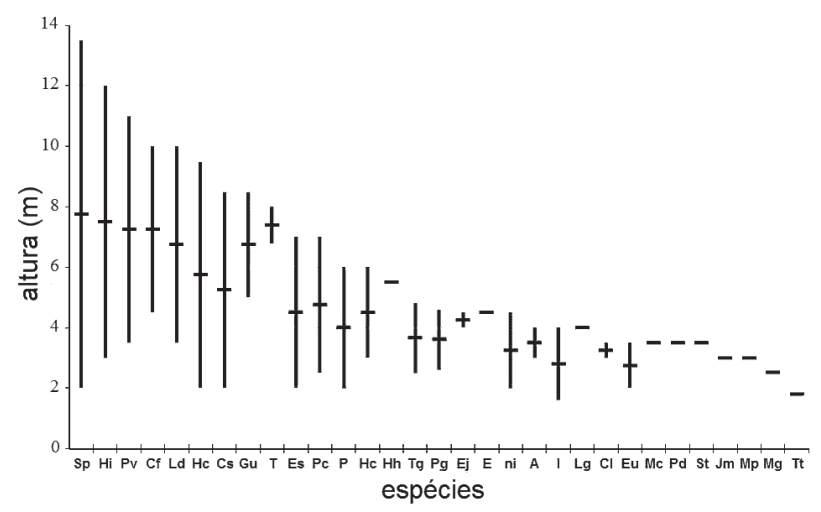

Figura 3. Valores máximos, médio e mínimo para a altura das espécies (abreviaturas na tabela 2) registradas no plantio heterogêneo em área da Mineradora Viterbo, São Paulo, SP, Brasil. Ver nomes completos das espécies na tabela 2.

Figure 3. Maximum, medium, and minimum height for the species (abbreviations in Table 2) recorded in the area of native tree plantation, Viterbo Machado Mining, São Paulo, São Paulo State, Brazil. View full names of the species in table 2. 
(2006), onze utilizaram o critério de inclusão de DAP $>4,7 \mathrm{~cm}$. Dentre estes, a área basal variou entre 9,23 e $52,78 \mathrm{~m}^{2} \mathrm{ha}^{-1}$ e a densidade de 1488,0 a $4463,3 \mathrm{~m}^{2} \mathrm{ha}^{-1}$. As áreas com maiores perturbações antrópicas apresentaram área basal inferior a $20,00 \mathrm{~m}^{2} \mathrm{ha}^{-1}$. Para uma área de mata ciliar recuperada no Município de Mogi Guaçu (Colmanetti et al. 2013), em um plantio também com nove anos de idade, a área basal foi de $18,29 \mathrm{~m}^{2} \mathrm{ha}^{-1} \mathrm{e}$ a densidade 808,9 ind ha $^{-1}$, também se adotando o critério de DAP $>4,7 \mathrm{~cm}$.

Para o período de tempo transcorrido, entre o plantio e o recenso após nove anos, o valor de área basal apresentado pode ser considerado alto. A reconstituição do solo é um dos processos mais lentos na recuperação de áreas degradadas (Silveira et al. 2006, Kitamura et al. 2008, Costa \& Zocche 2009). Em dez anos, áreas sob recuperação natural sob roças em pousio na Mata Atlântica apresentam de 8 a $14 \mathrm{~m}^{2}$ ha $^{-1}$ de área basal (Sampaio 1997). As condições de solo e a matriz nos quais se encontram são muito diferentes de uma cava de areia recuperada, mas apontam para resultados satisfatórios na restauração de uma estrutura florestal mínima na área de estudo.

O número total de espécies também é maior do que em outras cavas de areia, avaliadas sete e nove anos depois, nos municípios de São Paulo e Taubaté (Almeida \& Sánchez 2005). Seis espécies apresentaram pelo menos um indivíduo com crescimento em altura acima de $1 \mathrm{~m} \mathrm{ano}^{-1}$. Destas, duas espécies foram regenerantes espontâneos - Schizolobium parahyba (Vell.) S.F.Blake e Petrea volubilis L. - com matrizes presentes em áreas próximas ao empreendimento. Estas espécies estão entre as mais abundantes e foram as mais adequadas para a restauração do local, com ressalva para a espécie exótica $P$. volubilis.

Algumas espécies bem-sucedidas em outras experiências de restauração ecológica, como Schinus terebinthifolius Raddi (Souza et al. 2001), não se destacaram entre as espécies utilizadas no presente estudo. A razão pode estar na qualidade das mudas utilizadas, na adaptação da espécie às condições locais de solo e clima ou a outros fatores que não puderam ser analisados nos limites deste estudo.

De maneira geral, para o curto tempo transcorrido desde a restauração, já há um estrato arbóreo desenvolvido; e para o restabelecimento de uma diversidade mínima para a área consideramos o plantio satisfatório. No entanto, numerosas espécies do plantio inicial não se estabeleceram e outras de regeneração espontânea foram registradas; dentre estas, a presença de espécies exóticas é preocupante pelos perigos que podem vir a trazer à biodiversidade.

Um programa de monitoramento amplo e de longo prazo para áreas restauradas é necessário por parte do poder público para o estabelecimento de parâmetros de restauração adequados a cada situação (tipo de perturbação/atividade) e região; e não de maneira apenas pontual em um ou outro local, de forma que se possam avaliar as diretrizes para esta prática. Parâmetros para etapas intermediárias do processo são necessários para monitoramento e avaliação. No presente estudo, por exemplo, uma espécie exótica que constitui uma ameaça em potencial à biodiversidade foi incluida no plano aprovado pelo órgão ambiental.

\section{Agradecimentos}

À Mineradora Viterbo Machado Luz, pela disposição da área e relatórios para a realização deste trabalho.

\section{Literatura citada}

Almeida, R.O.P.O. \& Sánchez, L.E. 2005. Revegetação de áreas de mineração: critérios de monitoramento e avaliação do desempenho. Revista Árvore 29: 47-54.

Bitar, O.Y. 1997. Avaliação da recuperação de áreas degradadas por mineração na região metropolitana de São Paulo. 1997. Tese de Doutorado, Universidade de São Paulo, São Paulo.

Braga, J.M.S. 2005. Relatório Anual de monitoramento Ambiental. Mineradora Viterbo, São Paulo.

Brancalion, P.H.S., Rodrigues, R.R., Gandolfi, S., Kageyama, P.Y., Nave, A.G., Gandara, F.B., Barbosa, L.M. \& Tabarelli, M. 2010. Instrumentos legais podem contribuir para a restauração de florestas tropicais biodiversas. Revista Árvore 34: 455-470.

Cabral Junior, M., Suslick, S.B., Obata, O.R., Sintoni, A. 2008. A mineração no Estado de São Paulo: situação atual, perspectivas e desafios para o aproveitamento dos recursos minerais. Geociências 27: 171-92.

Colmanetti, M.A.A. \& Barbosa, L.M. 2013. Fitossociologia e estrutura do estrato arbóreo de um reflorestamento com espécies nativas em Mogi-Guaçu, SP, Brasil. Hoehnea 40: 419-435.

Costa, S. \& Zocche, J.J. 2009. Fertilidade de solos construídos em áreas de mineração de carvão na região sul de Santa Catarina. Revista Árvore 33: 665-674.

Devide, A.C.P., de Castro, C.M., Ribeiro, R.D.L.D., de Souza Abboud, A.C., Pereira, M.G. \& Rumjanek, N.G. 2014. História Ambiental do Vale do Paraíba do Sul, Brasil. Revista Biociências 20: 12-29.

Durigan, G., Engel, V.L., Torezan, J.M., Melo, A.C.G. de, Marques, M.C.M., Martins, S.V., Reis, A. \& Scarano, 
F.R. 2010. Normas jurídicas para a restauração ecológica: uma barreira a mais para dificultar o êxito das iniciativas? Revista Árvore 34: 471-485.

Fidalgo, O. \& Bononi, V.L.R. 1989. Manual prático de coleta, herborização e preservação. Instituto de Botânica do Estado de São Paulo, São Paulo.

Forzza, R.C., Leitman, P.M., Costa, A.F., Carvalho Jr, A.A., Peixoto, A.L., Walter, B.M.T., Bicudo, C., Zappi, D., Costa, D.P., Lleras, E., Martinelli, G., Lima, H.C., Prado, J., Stehmann, J.R., Baumgratz, J.F.A., Pirani, J.R., Sylvestre, L., Maia, L.C., Lohmann, L.G., Queiroz, L.P., Silveira, M., Coelho, M.N., Mamede, M.C., Bastos, M.N.C., Morim, M.P., Barbosa, M.R., Menezes, M., Hopkins, M., Secco, R., Cavalcanti, T.B. \& Souza, V.C. 2010. Introdução. In: Lista de Espécies da Flora do Brasil. Jardim Botânico do Rio de Janeiro, pp. 21-60.

Gomes, E.P.C., Sugiyama, M., Adams, C., Oliveira Jr., C.J.F. \& Prado, H.M. 2013.A sucessão florestal em roças em pousio: a natureza está fora da lei? Scientia Forestalis (IPEF) 41: 343-352.

Kitamura, A.E., Alves, M.C., Suzuki, L.G.A.S. \& Gonzalez, A.P. 2008. Recuperação de um solo degradado com a aplicação de adubos verdes e lodo de esgoto. Revista Brasileira de Ciência do Solo 32: 405-416.

Knapik, P. \& Maranho, L.T. 2008. Avaliação da revegetação em área de mineração, região de inundação do rio Iguaçu, Araucária, PR. Revista Brasileira de Biociências 5(S1): 507-509.

Lelles, L.C.D., Silva, E., Griffith, J.J. \& Martins, S.V. 2005. Perfil ambiental qualitativo da extração de areia em cursos d'água. Revista Árvore 293: 439-444

Marinho, N.F., Caproni, A.L., Franco, A.A., \& Berbara, R.L.L. 2004. Respostas de Acacia mangium Willd e Sclerolobium paniculatum Vogel a fungos micorrízicos arbusculares nativos provenientes de áreas degradadas pela mineração de bauxita na Amazônia. Acta Botanica Brasilica 18: 141-149.

Mechi, A. \& Sanches, D.L. 2010. Impactos ambientais da mineração no Estado de São Paulo. Estudos Avançados 24: 209-220.

Mueller-Dumbois, D. \& Ellenberg, H. 1974. Aims and methods of vegetation ecology. John Wiley \& Sons, New York.

Nappo, M.E.; Griffith, J.J.; Martins, S.V.; Junior, P.M.; Souza, L.; Oliveira Filho, A.T. 2004. Dinâmica da estrutura fitossociológica da regeneração natural em sub-bosque de Mimosa scabrella Bentham em área minerada, em Poços de Caldas, MG. Revista Árvore. 28: 811-829.

Nobre Filho, P.A., Sabadia, J.A.B., Duarte, C.R., Magini,
C., Neto, J.D.A.N. \& da Silva Filho, W.F. 2011. Impactos ambientais da extração de areia no canal ativo do Rio Canindé, Paramoti, Ceará. Revista de Geologia 24: 126-135.

Ogata, H. \& Gomes, E.P.C. 2006. Estrutura e composição da vegetação no Parque CEMUCAM, Cotia, SP. Hoehnea 33: 1-25.

Padmalal, D. \& Maya, K. 2014. River Sand Mining and Mining Methods. In: D. Padmalal \& K. Maya. 2014. Sand Mining, Springer, Dordrechet, pp. 23-30.

Pinheiro, R.A., Fisch, S.T.V. \& de Almeida, A. 2008. A cobertura vegetal e as características do solo em área de extração de areia. Revista Biociências 10: 103-110.

Richardson, D.M., Pyšek, P., Rejmánek, M., Barbour, M.G., Panetta, F.D. \& West, C.J. 2000. Naturalization and invasion of alien plants: concepts and definitions. Diversity and distributions 6: 93-107.

Rodrigues, R.R. \& Gandolfi, S. 1998. Restauração de florestas tropicais: subsídios para uma definição metodológica e indicadores de avaliação e monitoramento. In: L. Dias, J.M Mello. Recuperação de áreas degradadas, UFV/SOBRADE, Viçosa, pp. 203-215.

Sampaio, P.D. 1997. Florística e Estrutura de Floresta Atlântica Secundária - Reserva Biológica Estadual da Praia do Sul, Ilha Grande - RJ. Dissertação de Mestrado, Universidade de São Paulo, São Paulo.

Secretaria do Meio Ambiente do Estado de São Paulo. 2008. Avaliação da recuperação ambiental da mineração de areia para aperfeiçoar os instrumentos de gestão: várzea do Paraíba do Sul - trecho Jacareí Pindamonhangaba. Secretaria de Estado do Meio Ambiente. Coordenadoria de Planejamento Ambiental; coordenação Lina Maria Aché, Sônia Aparecida Abissi Nogueira e Neide Araújo; equipe técnica executora Antônio José Dias et al. SMA, São Paulo.

Silveira, R.B., Melloni, R. \& Melloni, E.G.P. 2006. Atributos microbiológicos e bioquímicos como indicadores da recuperação de áreas degradadas, em Itajubá/MG. Cerne 12: 48-55.

Souza, P.A., Venturin, N., de Macedo, R.L.G., Alvarenga, M.I.N., Silva, V.F. 2001. Estabelecimento de espécies arbóreas em recuperação de área degradada pela extração de areia. Cerne 7: 43-52.

Wanderley, M.G.L., Shepherd, G.J., Martins, S.E., Estrada, T.E.M.D., Romanini, R.P., Koch, I., Pirani, J.R., Melhem,T.S., Giulietti-Harley, A.M., Kinoshita, L.S., Magenta, M.A.G., Wagner, H.M.L., Barros, F., Lohmann, L.G., Amaral, M.C.E., Cordeiro, I., Aragaki, S., Bianchini, R.S. \& Esteves, G.L. 2011. Checklist das Spermatophyta do Estado de São Paulo, Brasil. Biota Neotropica 11: 193-390. 